\title{
EDITORIAL
}

\section{SOBRE LA REVISTA DE SANIDAD E HIGIENE PÚBLICA}

\author{
Gerardo Clavero González
}

He recibido de Cristina Pérez Andrés, Coordinadora de redacción, el encargo de escribir unas notas sobre la «Revista» (así la designaré en lo sucesivo). Dicha tarea me ha llenado de agradecimiento y preocupación. Estoy escribiendo en este número con amigos, compañeros y colegas sobre una teoría y práctica, una filantropía científica dedicada a promover la salud de personas y grupos sociales, especialmente de los mas necesitados. El agradecimiento ya ha sido expresado pero la preocupación persiste. ¿Hablo de la historia de la Revista o meditamos sobre la materia de la que trata, es decir la historia de la Salud Pública, en este caso, española?

Comienzo por un momento histórico de la Salud Pública Española relatando una anécdota de la juventud de Cajal. Cuenta que quizá en 1878 me enteré de que en la calle del León, número 25 principal Don Francisco Chenel proporcionaba a plazos excelentes microscopios. Revivamos el hecho descrito: Casi convaleciente de paludismo cubano, Cajal se acerca a un buen microscopio. Considero que aquí nace además del histólogo el gran sanitario que fue desde este momento, a través de la valoración internacional que obtuvo como Premio Nobel deriva la Salud Pública Española en gran medida.

Un segundo momento histórico es 1898 y un resultado próximo el nacimiento de la política social en España y el renacimiento económico español (Vicens Vives). Para nos- otros cambió la vida de Cajal (enfermedad vs actuación sanitaria) y ahí surgió el mejor impulso de la sanidad del país. Dos años después se creó el Ministerio de Trabajo y, más tarde, en su seno el Instituto Nacional de Previsión (1908). La Redacción de esta Revista permitiéndonos incluir las copias de las portadas de algunos libros nos posibilita exponer la de la Legislación Sanitaria de Seguridad Social, Tomo 111 (figura 1) que contiene ordenada y extensamente las disposiciones jurídicas en sanidad pública hasta el 31 de diciembre de 1954. En el Instituto Nacional de Previsión se realizaron estudios preparatorios entre 1931-1936 para, de acuerdo con el ordenamiento internacional, implantar a corto plazo en España el seguro de enfermedad.

El tercer momento es 1918. En esta fecha se produjo la epidemia de gripe (la «española») (figura 2) y, como resultado probable, la estatalización de los médicos rurales en España. Esta modificación de la medicina rural desde lo privado a lo público se complementó cuando a los sanitarios locales ya designados como funcionarios se les otorgó autoridad municipal. Surgió entonces un alto número de colaboradores y lectores para una futura Revista de Sanidad.

La cuarta etapa fue la creación de las Escuelas Nacionales de Sanidad. Se reproduce la cubierta y el contenido del libro de C. Prausnitz (1933) (figura 3) donde se recoge 
la «movida docente» provocada por la Sociedad de Naciones y las creaciones europeas.

Este momento da pie para citar la creación de la Escuela Nacional de Sanidad en el Instituto Nacional de Sanidad Alfonso XIII y también la lucha antipalúdica, ambas dirigidas por Don Gustavo Pittaluga, personalidad eximia que padeció el exilio provocado por la guerra civil. La creación de la Escuela hizo más necesario el mantenimiento de la Revista, fundadas ambas en el mismo año.

La Revista se creó por Real Orden del Ministerio de la Gobrenación de 12 de noviembre de 1925. disponiendo que en sustitución del Anuario de la Dirección General de Sanidad, instituido por Real Orden de 26 de noviembre de 1920, se cree una publicación bimestral entonces que se denominará Boletín Técnico de la Dirección General de Sanidad.

La información sobre la Revista en este período desde 1926 hasta 1936 resulta difícil de encontrar ya que las bibliotecas son discretamente accesibles y el tiempo del que he dispuesto ha sido muy escaso. Sí me ha sido más fácil encontrar la legislación de la época, aunque parcialmente. Ello da idea de las Instituciones, pero muchos aspectos funcionales solo podrán conocerse con datos administrativos y económicos de obtención laboriosa.

Ciertas publicaciones oficiales de la época están en mi poder; por ello, conforme con el aforismo de que «una imagen vale más que mil palabras» la Secretaría de Redacción de la Revista me permite que se añada a estas breves líneas las primeras páginas de algunas publicaciones oficiales por las que pueden conocerse cuáles eran los problemas sanitarios de aquélla época y sus soluciones (figuras 4-10). Quiero destacar entre ellas la portada del libro El paludismo en el campo, de Sadi de Buen, del Cuerpo Médico de Sanidad Nacional, que investigó sobre paludismo y fiebre recurrente y descubrió el vector de esta útima enfermedad. Fue fusilado durante los primeros días del golpe militar de 1936.

Para terminar debo proponer una alternativa para facilitar un análisis en profundidad sobre la Salud Pública Española en un período que considero «de oro», en paralelo con la aportación que la Revista de Sanidad e Higiene Pública hizo al Congreso Nacional de Sanidad en 1934 y es la siguiente: en 2009 se cumplirá el 75 aniversario del único Congreso Nacional de Sanidad que se ha celebrado en España, y estando tan cerca su 75 aniversario, las sociedades científicas deberían convocar una celebración onomástica con la especial participación de cátedras y sociedades de higiene y salud pública y con las de historia de la medicina. Se recuerda que el secretario general del Congreso Nacional de Sanidad de 1934 fue el profesor Don Luis Nájera Angulo, y resulta obligado ensalzar su recuerdo. Estamos seguros de que en dicho $2^{\circ}$ Congreso se valorarían cumplidamente las actividades sanitarias de la época. 


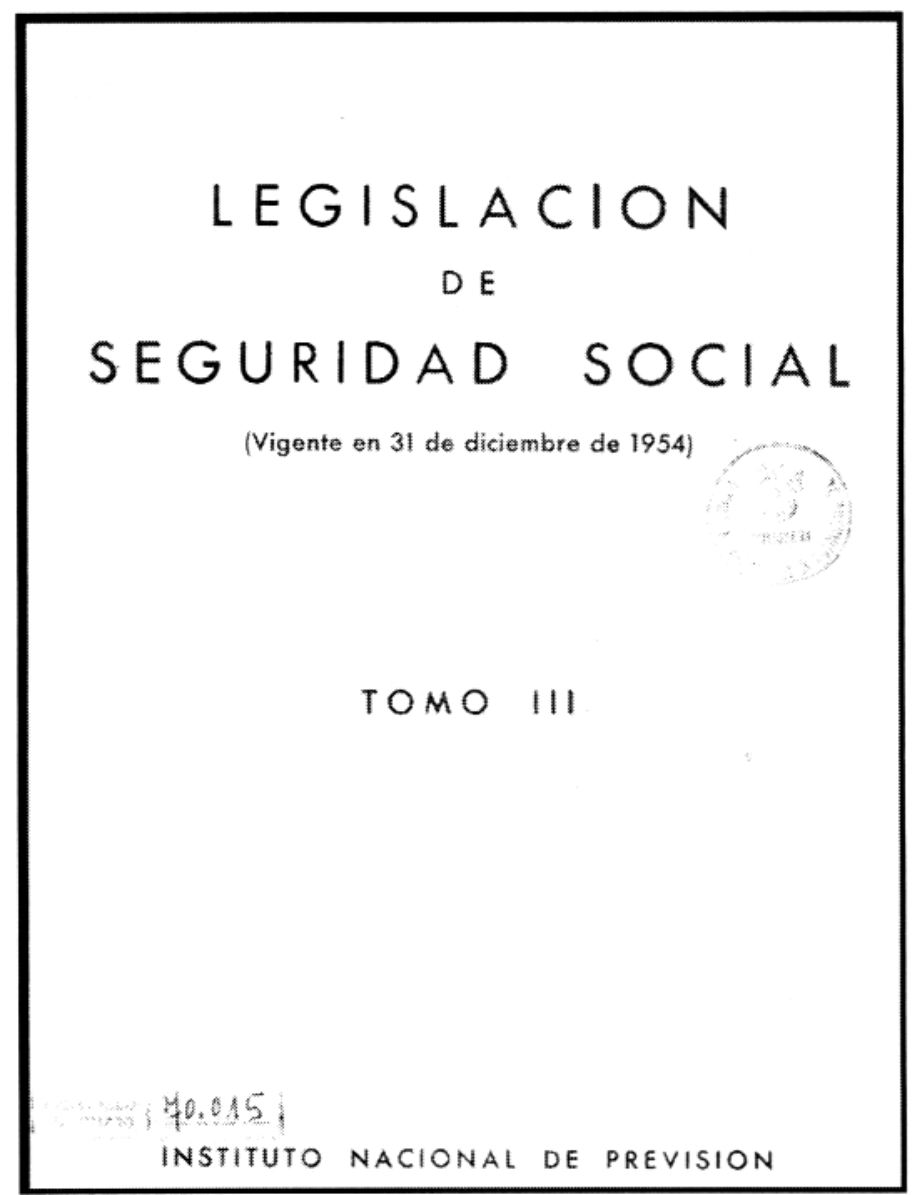

FIGURA 1 


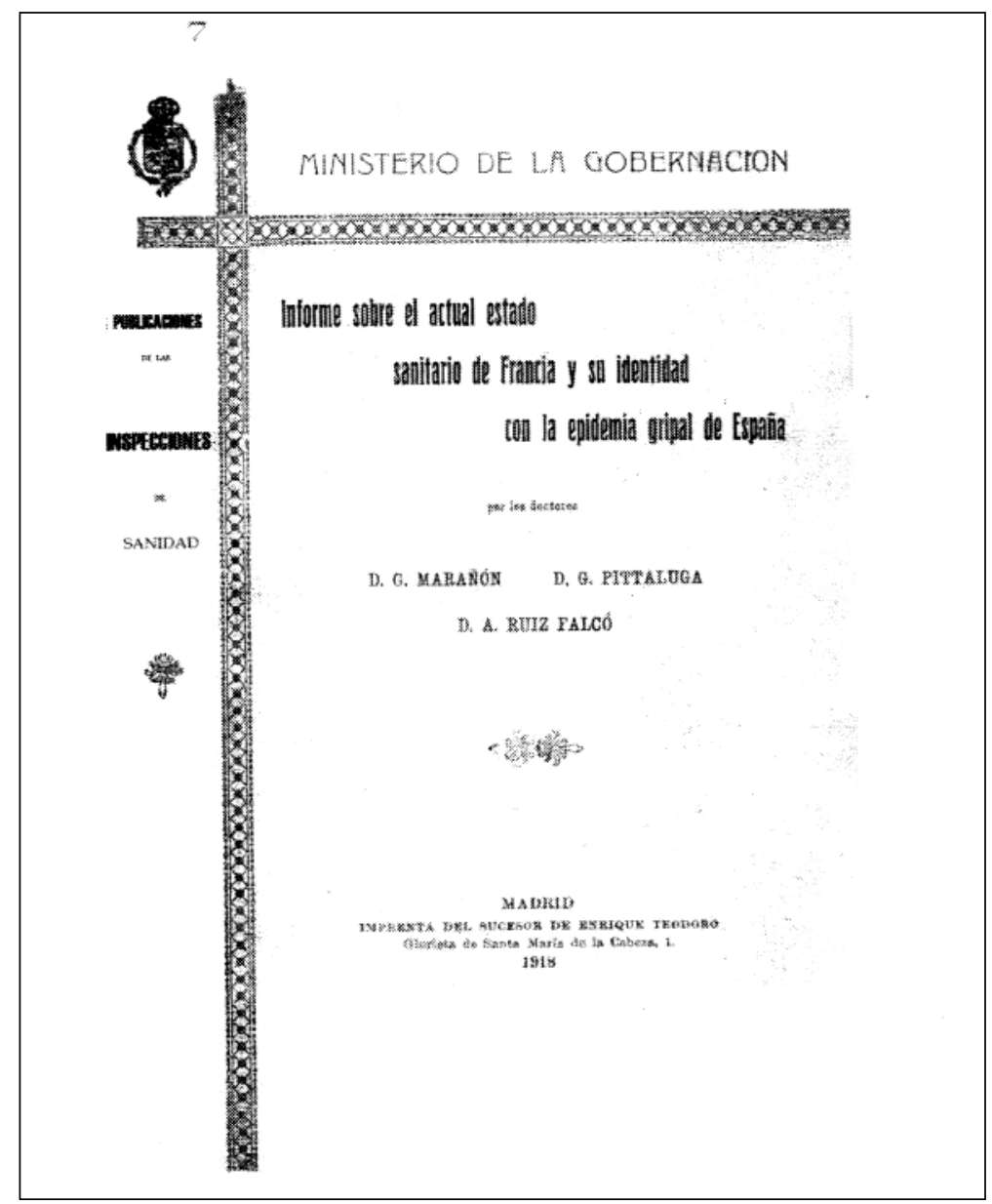

FIGURA 2 
UNIVERSITY OF LONDON EREATH CLARK LECTVXXSS, T9Y3 delivered at

The Londos Sthow of Hygiene and Tropical Medicine

\section{The Teaching of Preventive Medicine in Europe}

\author{
By
}

CARL PRAUSNITZ

W.D. (Bresliew), M.R.C.S. (Eng.) L.R.C.P. (1.and.) Prefersor of Hyziene in the Limikersity af Brestan

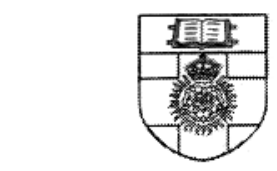

OXFORD UNIVERSITY PRESS LONDON : HUMPHREY MILFORD $x 933$

\section{CONTENTS}

LECTUR:

1. INTRODUCTION .

I. THE TEACHING OF PREVENTIVE MEDICINE IN FRANCE

III. THE TEACHING OF PREVENTIVE MEDICINE IN GERMANY

VV. THE TEACHING OF PREVENTTVE MEDICINE IN GREAT BRITAIN . . . THE TEACHING OF PREVENTIVE MEDICINE IN POLAND .

VI. THE TEACHING OF PREVENTIVE MEDICINE IN HUNGARY . . 89 VII. THE TEACHING OF PREVENTIVE MEDICINE IN CZECHOSLOVAKIA. . . . IO VIII. THE TBACHING OF PREVENTIVE MEDICINE IN YUGOSLAVIA. . . . . . . $\times 19$ IX. THE TEACHINO OF PREVENTIVE MEDICINE IN SPALN . . . . . 141 $X$. THE TEACHING OF PREVENTIVE MEDICINE IN GREECE . . . . . . . 149 XI. THE TEACHING OF PREVENTIVE MEDICINE IN RUSSIA . . . . . . 253 XII. THE WORK OF THE LEAGUE OF NATIONS + 267 INDEX OF NAMES . . . . . . . 174 SUBJECT INDEX. . . . . . . 275

FIGURA 3 


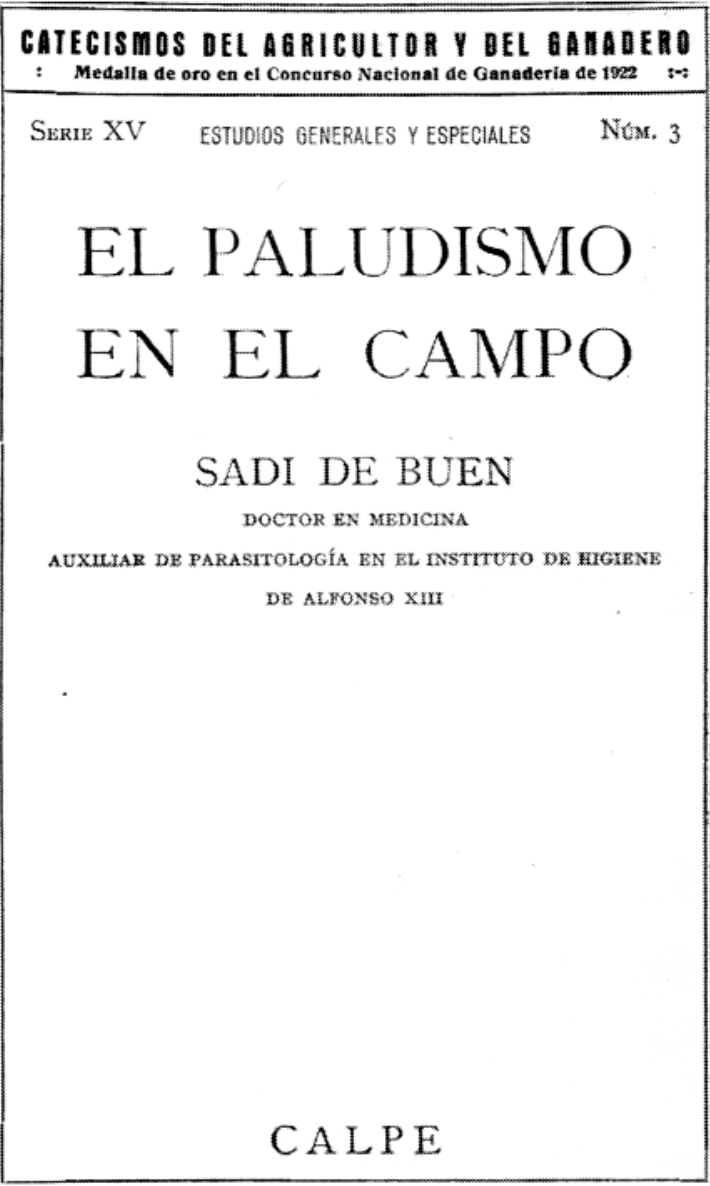

FiguRA 4 


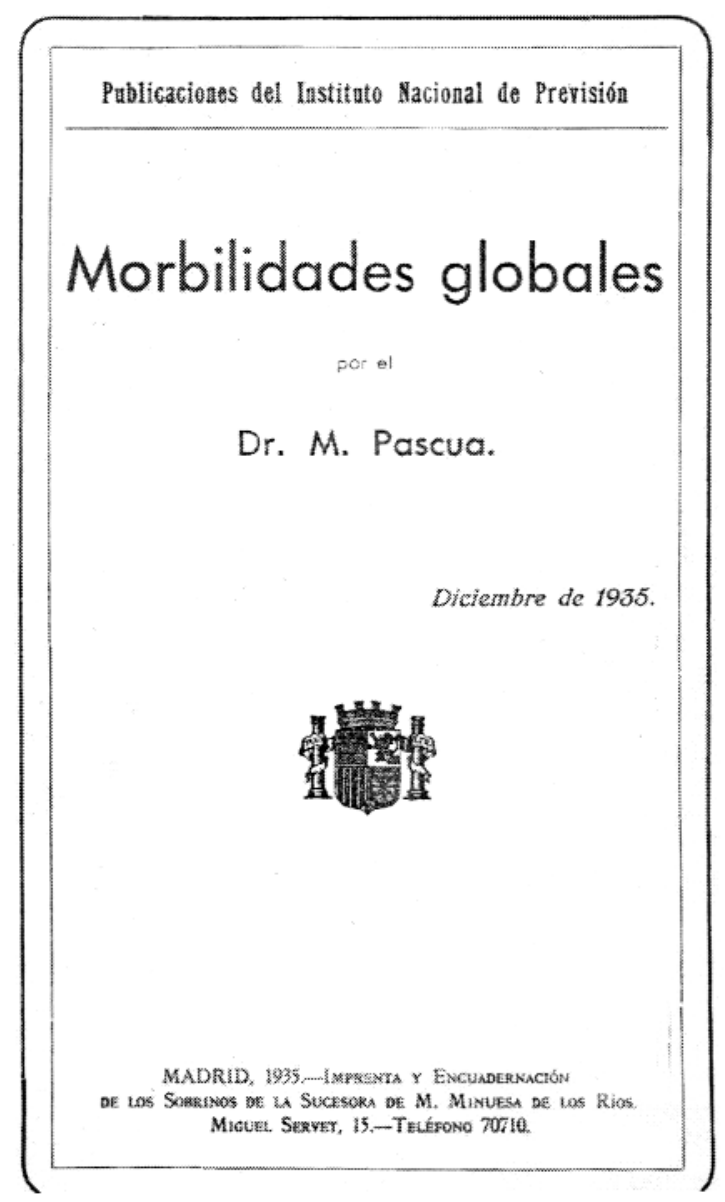

FIGURA 5 
Publicaciones del Instituto Nacional de Previsión

Unificación de los Seguros sociales.

\section{Compilacion de dispositiones levisidutinas extrmujerns}

sobre

\section{Seguro de Enfermedad. \\ TOMO SEGUNDO}

butgaria, CHECOSLOVAQUiA, CHILE, DINAMARCA $Y$ FRANCIA

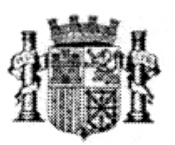

MADRID, 1933.-IMPRENTA Y ENCUADERNACIÓN de tos Solsrinos de LA Sucesora dE M. Minuesa de los Rlos. Migunl Servet, 15,-Thiźfono 70710.

FIGURA 6 


\title{
REGLAMENTO ORGANICO \\ DE \\ SANIDAD EXTERIOR
}

\author{
APROBADO POR DRCRETO DEL, MINISTERIO \\ DB TRABAJO, SANIDAD Y FURYSION DE 7 \\ DE SEPTREMBRÉ
}

I 934

FIGURA 7 


\section{SANIDAD NACIONAL \\ CENTROS \\ DE. \\ HIGIENE RURAL \\ DE LA \\ Provincia de Sartander \\ pOR LOS DOCTORES \\ G. CLAVERO DEL CAMPO \\ INSDECTOR DEOVINCIAI DE 5 MNIDAD \\ ANGOLOTI Y DIAZ FLÓREZ \\ DIRECTORES DE LOS CENTROS DE HIGTENE \\ DE REINOSA Y SANTONA}

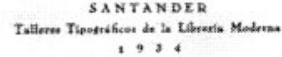

FIGURA 8 


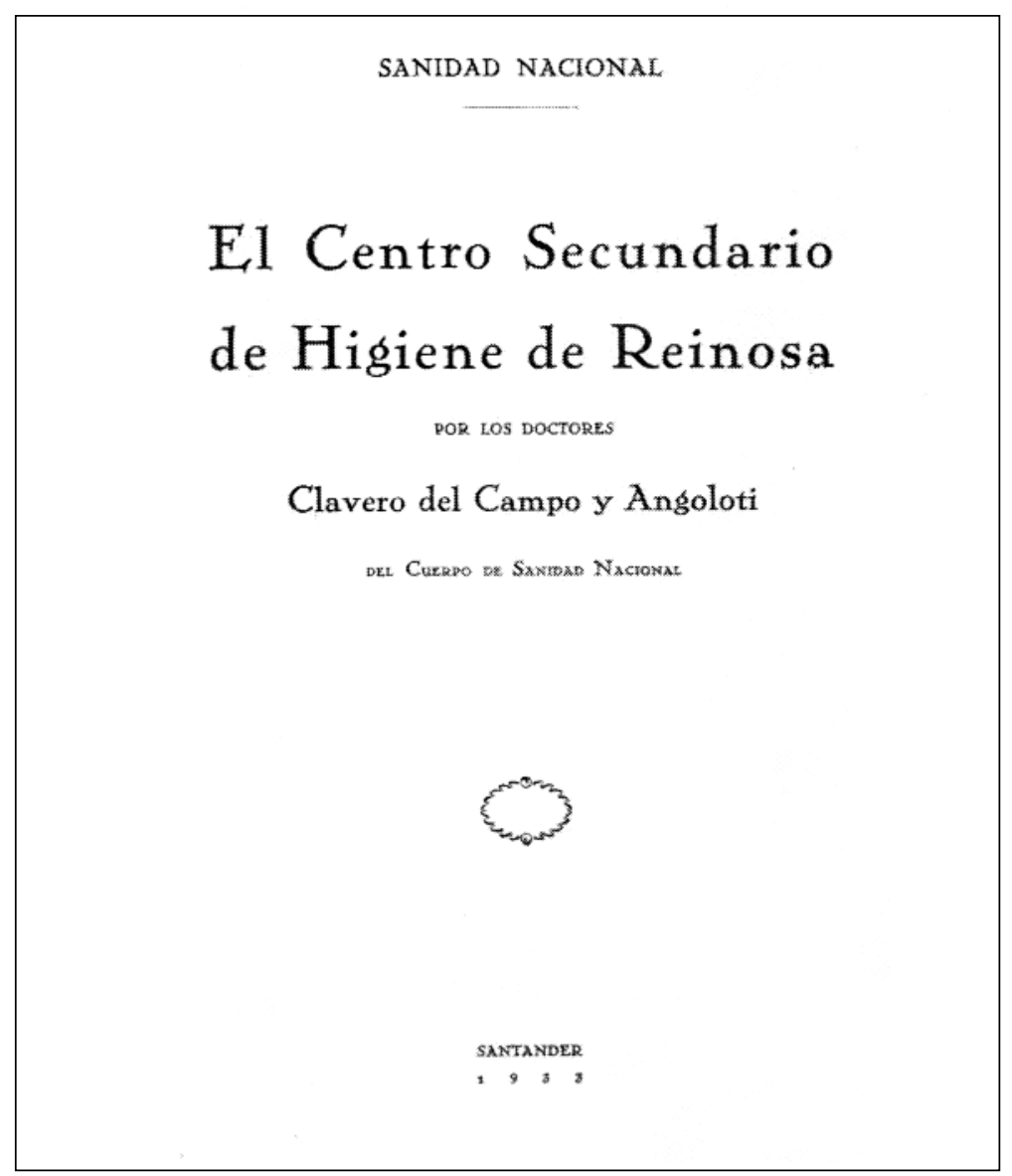

FIGURA 9 


\section{TUBERCULOSIS Y VACUNA B. C. G. \\ POR E}

D. J. J. LASTRA

DEL INSTITUTO PROVINCIAL DE HIGIENE

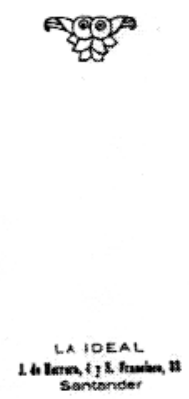

FIGURA 10 